\title{
Catalytic Solid-Phase Seeding of Silicon Nanowires by Nickel Nanocrystals in Organic Solvents
}

\author{
Hsing-Yu Tuan, Doh C. Lee, Tobias Hanrath, and Brian A. Korge/* \\ Department of Chemical Engineering, Texas Materials Institute, \\ Center for Nano- and Molecular Science and Technology \\ The University of Texas at Austin, Austin, TX 78712-1062
}

* Corresponding author: Phone: (512) 471-5633; FAX: (512) 471-7060; email: korgel@mail.che.utexas.edu

As described below, Si nanowires were synthesized in a semibatch high pressure $1 \mathrm{~mL}$ grade 2 titanium reactor cell configured for reactant injection at high temperature and pressure. Stock solutions of silane precursors with sterically-stabilized $\mathrm{Ni}$ nanocrystals were pre-mixed and then injected at the desired temperature and pressure using a high pressure liquid chromatography (HPLC) pump to control the injection rate and the reactor pressure.

\section{Ni nanocrystal synthesis}

$\mathrm{Ni}$ nanocrystals were prepared by nickel carbonyl reduction following procedures developed by Murray and coworkers. ${ }^{1}$ At room temperature, $1 \mathrm{mmol}$ of $\mathrm{Ni}\left(\mathrm{CH}_{3} \mathrm{COO}\right) \cdot 4 \mathrm{H}_{2} \mathrm{O}$ (Aldrich) was mixed with $0.5 \mathrm{mmol}$ of oleic acid (Aldrich), $2 \mathrm{mmol}$ of trioctylamine (Aldrich) and $0.25 \mathrm{mmol}$ of trioctylphosphine (Fluka) in $10 \mathrm{~mL}$ of diphenylether (Aldrich) in a three-neck flask. The mixture was agitated at room temperature while flushing with nitrogen for $\sim 20 \mathrm{~min}$. After heating the solution to $200^{\circ} \mathrm{C}, 0.5 \mathrm{mmol}$ of trioctylphosphine was injected. At this point, the green solution 
becomes a dark-green color. The mixture was then heated to $250^{\circ} \mathrm{C}$. Separately, $0.5 \mathrm{~g}$ 1,2-hexadecanediol (Aldrich) was dissolved in $2.5 \mathrm{~mL}$ of diphenylether and heated to $80^{\circ} \mathrm{C}$ under nitrogen atmosphere. The diol solution was injected into the nickel carbonyl/phosphine solution once it reached $250^{\circ} \mathrm{C}$. The solution temperature immediately dropped to $\sim 220^{\circ} \mathrm{C}$ after injection, and was raised back to $250^{\circ} \mathrm{C}$. The mixture was held at $250^{\circ} \mathrm{C}$ for 20 min with agitation. The nanocrystals were removed from heat and allowed to cool to room temperature. The solution was collected and centrifuged at $8000 \mathrm{rpm}$ for $10 \mathrm{~min}$. A small amount of poorly capped particles precipitated and was discarded. The well-dispersed nanocrystals were then mixed with $20 \mathrm{~mL}$ of ethanol. The hydrophobic Ni nanocrystals flocculated and were collected by centrifugation at $8000 \mathrm{rpm}$ for $10 \mathrm{~min}$. The supernatant was discarded. After an additional rinse with ethanol, the purified Ni nanocrystals were collected and dried on a rotary evaporator. The nanocrystals could be redispersed readily in hexane. The nanocrystals were stored in a nitrogen-filled glove box until needed. Fig. 2 shows a TEM image of Ni nanocrystals with a diameter of $5.59 \pm 0.59 \mathrm{~nm}$ used to seed $\mathrm{Si}$ nanowire synthesis.

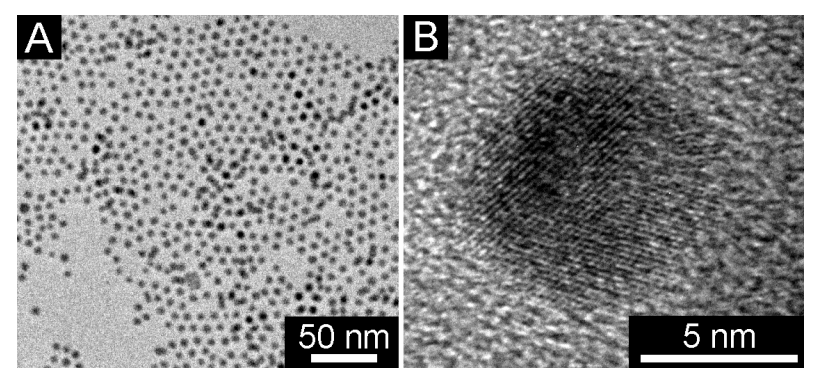

Figure 1. TEM images of Ni nanocrystals used to seed $\mathrm{Si}$ nanowire synthesis. The average nanocrystal diameter is $5.59 \pm 0.59 \mathrm{~nm}$. 


\section{Reactor Design}

The experimental setup is similar to the one designed by Hanrath and Korgel. ${ }^{2} \mathrm{~A}$ 1-ml grade 2 titanium reactor is connected to $1 / 16$ " od 0.03 " id stainless steel highpressure tubing (High Pressure Equipment; HIP) via grade-2 titanium LM6-HIP reducers (HIP) and stainless steel high-pressure valves as shown in Figure 2 (HIP). The inlet was connected to a 6-way valve (Valco) with a 340 $\mu$ injection loop injection loop. The outlet was connected to a micrometer valve (HIP). The reactor is pressurized using a high-pressure liquid chromatography (HPLC) pump (Alcott) connected to a piston filled with anhydrous solvent. The piston is pressurized using water to avoid running solvent directly through the pump. The reactor pressure was monitored using a digital pressure gauge (Sensotech). The reactor was placed in a brass heating block with a thermocouple to measure temperature, connected to an Omega temperature controller to maintain the reaction temperature.

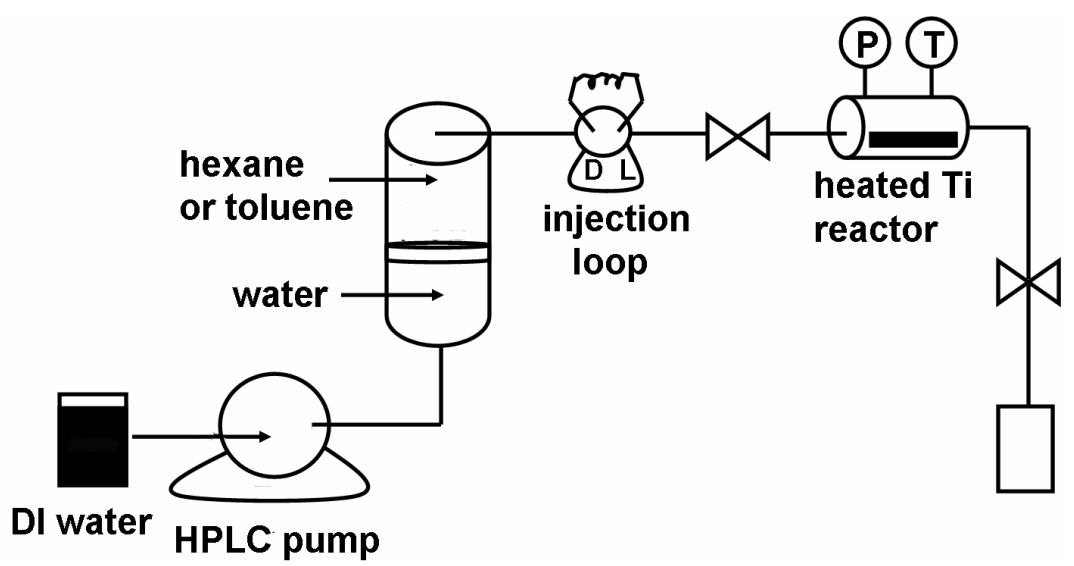

Figure 2. Schematic of the high pressure reactor system used for nanowire synthesis in a supercritical fluid.

\section{Nanowires synthesis}


Anhydrous toulene, hexane, and monophenylsilane (MPS; $\left.\left(\mathrm{C}_{6} \mathrm{H}_{5}\right) \mathrm{H}_{3} \mathrm{Si}\right)$ were purchased from Sigma-Aldrich, n-octylsilane $\left(\left(\mathrm{C}_{8} \mathrm{H}_{17}\right) \mathrm{H}_{3} \mathrm{Si}\right)$ was purchased from Gelest, and trisilane $\left(\mathrm{Si}_{3} \mathrm{H}_{8}\right)$ was purchased from Voltaix. All chemicals were used as received. Ni nanocrystals were synthesized as described above. Stock solutions of $80 \mathrm{mM}$ MPS, octylsilane, and trisilane, in toluene or hexane were mixed with Ni nanocrystals at the desired $[\mathrm{Ni}] /[\mathrm{Si}]$ mole ratio in a nitrogen-filled glove box.

A silicon wafer cut to $4 \times 30 \mathrm{~mm}$ and ultrasonically cleaned in acetone for $30-\mathrm{min}$ was inserted into the reactor to help collect the nanowire product. Before injecting the reactants, the reactor is heated to the desired reaction temperature and pressurized to 3.4 $\mathrm{MPa}$. The stock solutions are then injected from a $340 \mu$ injection loop, which raised the pressure to $\sim 20 \mathrm{MPa}$. The reaction was allowed to proceed for $10 \mathrm{~min}$. The reactor was then cooled by removing the insulation materials and turning off the heating element. Once the reactor cooled to room temperature, the reactor was flushed with hexane and the soluble product was collected in a vial. The deposition substrate was coated with a grey material and was removed from the reactor. The reaction products were stored in a nitrogen-filled glove box prior to characterization.

\section{Materials Characterization}

The silicon nanowires were characterized by high-resolution transmission electron microscopy (HRTEM), high-resolution scanning electron microscopy (HRSEM) and energy dispersive x-ray spectroscopy (EDS). HRSEM images were obtained on a LEO $1530 \mathrm{HRSEM}$ operating between $1.5 \mathrm{kV}$ to $5 \mathrm{kV}$ with working distance between $2 \mathrm{~mm}$ to $7 \mathrm{~mm}$ using an in-lens detector to study the morphology of nanowies on the silicon 
substrate. HRTEM images and EDS data were obtained using a JEOL 2010F TEM at operating voltage $200 \mathrm{KV}$ with attached Oxford INCA EDS and images were obtained by a GATAN digital photography system. The HRTEM sample was made by scratching the surface of the silicon substrate with a lacey carbon-coated TEM grid (Electron Microscopy Sciences).

\section{References}

(1) Murray, C. B.; Sun, S.; Doyle, H.; Betley, T., MRS Bull. 2001, 26, 985-991.

(2) Hanrath, T.; Korgel, B. A., J. Am. Chem. Soc. 2002, 124, 1424-1429. 